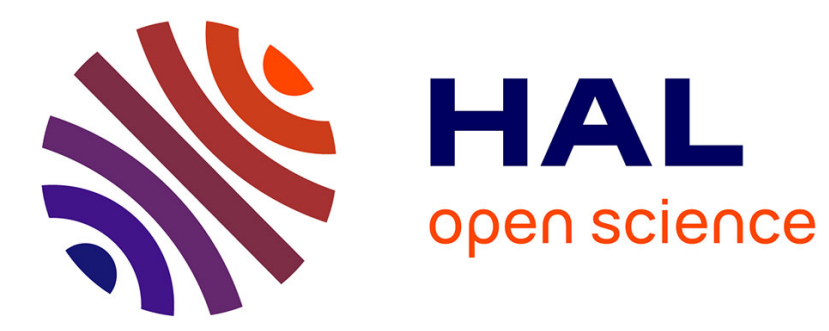

\title{
Les GAP comme vecteurs de changements
}

\author{
Nicolas Hervé, Cécile Gardiès
}

\section{To cite this version:}

Nicolas Hervé, Cécile Gardiès. Les GAP comme vecteurs de changements. J.-F. Marcel (Ed.) Lycées agricoles en changements, Educagri Editions (collection Agora recherche), pp.127-143, 2014, 2844449786. halshs-02369211

\section{HAL Id: halshs-02369211 https://shs.hal.science/halshs-02369211}

Submitted on 18 Nov 2019

HAL is a multi-disciplinary open access archive for the deposit and dissemination of scientific research documents, whether they are published or not. The documents may come from teaching and research institutions in France or abroad, or from public or private research centers.
L'archive ouverte pluridisciplinaire HAL, est destinée au dépôt et à la diffusion de documents scientifiques de niveau recherche, publiés ou non, émanant des établissements d'enseignement et de recherche français ou étrangers, des laboratoires publics ou privés. 


\title{
Les GAP comme vecteurs de changements
}

\author{
Nicolas HERVÉ* \\ Cécile GARDIES *
}

\section{Introduction :}

Nous nous intéressons dans ce chapitre à un dispositif institutionnel, mis en place par le Ministère de l'Agriculture, de l'Agroalimentaire et de la Forêt (MAAF), afin de faire évoluer son système de formation continue des enseignants.

Les pratiques des enseignants évoluent au gré à la fois des contextes, que l'on peut qualifier actuellement de mouvants tant du point de vue de la formation initiale que de la formation continue, ainsi que des changements institutionnels qui modifient les tâches prescrites, par exemple au travers des rénovations successives des programmes de formation ou bien en assignant des tâches de coordination ou de suivis individuels aux enseignants. Par ailleurs un certain nombre d'entre eux sont mobilisés pour participer à la formation de leurs pairs, que ce soit comme conseiller pédagogique accompagnant l'entrée dans le métier, ou encore comme formateur occasionnel (en temps ou en thématiques) dans l'accompagnement à la construction de nouvelles compétences pour des enseignants en cours de carrière. Les changements induits par cette évolution dans leur fonction n'est pas sans interroger les liens entre leur pratique et les savoirs nécessaires à ces évolutions dans de nouveaux modèles de développement professionnels.

* Docteur en Didactique de la Physique, UMR EFTS, Université de Toulouse, ENFA.

* Maître de conférences (HDR) en Sciences de l'information et de la communication, UMR EFTS, Université de Toulouse, ENFA. 
Nous nous penchons sur les questions soulevées par ce changement à partir de l'exemple des GAP (Groupes d'Animation et de Professionnalisation), dispositif innovant d'accompagnement à la professionnalisation des enseignants de l'Enseignement Agricole qui constituent actuellement une réponse institutionnelle à la fois à des changements dans la politique de formation continue des enseignants, et à la prise en compte de questions «vives» dans les dimensions d'enseignement/apprentissage de l'activité de l'enseignant. Nous nous intéressons tout particulièrement ici au rôle accordé à l'analyse didactique des pratiques d'enseignement au sein de ces GAP, en tant qu'outil de développement professionnel des enseignants dans une visée de changement de posture vers un rôle de formateur.

Ce travail situe le changement étudié dans une planification au sein de l'organisation, élaboré et mis en œuvre par ses acteurs au travers d'un projet qui vise sa transformation. Il l'aborde dans l'axe épistémologique, c'est à dire celui des connaissances et des savoirs, de la culture de l'organisation notamment dans les nouveaux modes d'organisation avec l'objectif d'élaborer de nouveaux savoirs mais aussi de modifier les modalités de leurs circulations, de leurs conversions ou de leurs capitalisations. Il convoque un plan onto-épistémologique, celui des rapports des sujets aux connaissances et à la culture de l'organisation.

Nous présentons dans un premier temps le contexte de la formation continue des enseignants, puis l'analyse des pratiques, en soulignant en particulier l'intérêt de fonder notre point de vue dans une perspective didactique, en interrogeant son rôle dans le développement professionnel et le changement de posture visé. Au travers de l'exemple des GAP nous analysons ce processus appuyé sur une analyse didactique instrumentée que nous mettons en perspective du changement attendu dans la formation continue. Notre orientation méthodologique s'appuie sur une démarche de recherche-intervention qui, au plus près des acteurs et en collaboration avec eux, va mobiliser la recherche pour élaborer des connaissances que ces acteurs pourront mettre à profit pour résoudre leur problème ou élaborer une réponse à leurs interrogations.

\section{Des changements dans la formation continue des enseignants de l'Enseignement Agricole}




\subsection{Un changement institutionnel dans l'organisation de la formation continue}

La politique portée par le Ministère de l'Agriculture en matière de formation continue des enseignants a évolué, notamment dans le cadre des

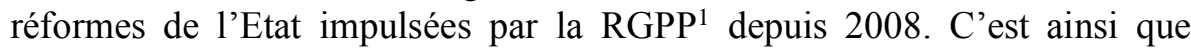
l'offre de formation, historiquement construite et organisée à l'échelle nationale, passe progressivement à un niveau régional. Le plan national de formation a par conséquent diminué en volume, en se recentrant sur certaines thématiques définies par la DGER ${ }^{2}$ comme prioritaires, et c'est le plan régional de formation qui se retrouve en capacité de financer des actions de formation, au plus près des besoins territoriaux, généralement sur une durée d'une journée.

Ce sont donc les DRAAF - $\mathrm{DAAF}^{3}$ qui financent et organisent maintenant une part de la formation continue des personnels enseignants, dans le but de limiter les coûts et les absences en établissement liés au transport des stagiaires sur de longues distances. A l'heure actuelle, le plan de formation est élaboré à partir d'un recensement local des besoins des personnels et des spécificités de leur territoire, et ce sont principalement les stages de formation disciplinaire qui sont organisés à cet échelon.

\subsection{Une réponse institutionnelle à ce changement : l'essor des GAP}

Pour répondre à ce « tournant organisationnel » (Malet \& Brisard, 2005), un dispositif se développe: les groupes d'animation et de professionnalisation (GAP).

Créés en 2002, les GAP étaient destinés à construire et mettre en œuvre des formations en régions, afin d'accompagner la prise de fonction des nombreux néo-titulaires issus des concours dits «Sapin». Les GAP, originairement appelés groupes d'animation pédagogique, étaient constitués d'une équipe d'enseignants reconnus experts par l'école de formation dans

Révision Générale des Politiques Publiques

Direction Générale de l'Enseignement et de la Recherche

Directions Régionales de l'Alimentation, de l'Agriculture et de la Forêt (DAAF

pour les territoires et départements d'outre-mer) 
l'enseignement de leur discipline et des formateurs d'une ENA ${ }^{4}\left(\mathrm{ENFA}^{5}\right.$, SupAgro Florac par exemple). Ce dispositif a été expérimenté de 2002 à 2005 autour de 3 thématiques (deux disciplinaires : $\operatorname{ESC}^{6}$ et Mathématiques ; une transversale: le paysage), sans qu'une extension du dispositif ne soit envisagée par la suite.

A partir de 2008, en prévision d'une réforme de la formation continue des personnels, le dispositif est relancé, il est financé par la DGER dans le cadre du $\mathrm{SNA}^{7}$ et change de nom : l'acronyme GAP reste mais le P de l'adjectif «pédagogique » devient le nom commun «professionnalisation». Depuis cette date, 12 GAP se développent, et sont majoritairement associés à une discipline scolaire (Documentation, Physique-Chimie, ESC, Mathématiques, EPS $^{8}$, Lettres, Agroéquipements, Agronomie, Zootechnie, Economiegestion, Histoire-géographie). Seul un GAP porte sur un thème transversal (Troubles Dyslexiques).

\subsection{Le GAP : un dispositif de formation d'enseignants « mixte »}

Le GAP est défini actuellement (note de service DGER/MSSI/N2013, 2039, SG/SRH/SDDPRS/N2013,1060 du 20 mars 2013) comme étant un dispositif d'accompagnement qui construit des réponses opérationnelles à des besoins repérés par l'école de formation et l'inspection et analysés dans l'Enseignement Agricole (veille et prospective sur une discipline ou thématique; production de ressources testées en classe ; sessions régionales ou inter-régionales de formation animées par les membres du GAP ; animation d'outils de diffusion des ressources: sites internet, bulletins imprimés, etc.).

Il se réunit deux fois par an en séminaires pour une durée totale annuelle de 5 jours. Des visioconférences ont également lieu entre les séminaires.

Etablissement national d'appui

Ecole Nationale de Formation Agronomique

Education Socioculturelle

Système National d'Appui

Education Physique et Sportive 
Le GAP est un dispositif de formation que l'on peut qualifier de « mixte », à la fois dans la composition de ses membres, et dans la manière dont y est envisagée la formation.

\subsubsection{Mixité statutaire des membres du GAP}

Un GAP réunit une dizaine d'enseignants autour d'une discipline ou d'une thématique pédagogique ou didactique. Chaque enseignant du GAP s'engage à faire partie du groupe pour au moins une année scolaire, mais l'idée est d'assurer une certaine continuité dans la mise en œuvre des projets définis. Ce collectif d'enseignants est encadré par des animateurs qui sont des formateurs ou des enseignants-chercheurs de l'ENFA de la discipline et/ou des sciences de l'éducation. Des inspecteurs de l'Enseignement Agricole peuvent également intervenir lors des séminaires. La diversité des membres d'un GAP nous autorise par conséquent à qualifier ce dispositif de «mixte».

\subsubsection{Mixité des objectifs et modalités de formation}

La formation des enseignants est envisagée à deux niveaux dans un GAP.

Tout d'abord, d'un point de vue intrinsèque au dispositif, le GAP est un outil de formation pour les membres du GAP eux-mêmes puisque ceux-ci vont être amenés à former leurs collègues dans des sessions de formation régionales ou inter-régionales. Ainsi, le premier lieu de formation est le GAP lui-même: il a pour objectif de professionnaliser ses membres afin qu'ils puissent être formateurs d'enseignants en région ou inter-régions.

Ensuite d'un point de vue extrinsèque au dispositif, des enseignants vont être formés aux productions produites par le GAP dans des sessions de formation d'une journée. Il s'agit alors pour le GAP de construire une ingénierie de formation adaptée à des sessions de formation d'une journée et permettant de diffuser les productions réalisées.

Un GAP relève par conséquent d'un dispositif de formation «mixte », qui met en œuvre différents types d'ingénierie. Du point de vue intrinsèque, puisque c'est un collectif qui se réunit pour échanger et construire collectivement des ressources et formations, que ces ressources sont testées en classe, il semble que l'ingénierie "naturelle » soit celle de l'analyse de pratiques. Du point de vue extrinsèque, puisque les journées de formation en 
région sont courtes ( 1 ou 2 journées), il semble au contraire que l'ingénierie privilégiée soit de type classique et transmissive.

Toutefois, la professionnalisation attendue des enseignants du GAP brouille les pistes «naturelles ». En effet, l'analyse de pratiques seule peutelle faire d'un enseignant un formateur d'enseignants, c'est-à-dire peut-elle généraliser son savoir pratique afin de le communiquer, et l'adapter hors du contexte dans lequel il a été créé ? Réciproquement, ne peut-on pas attendre d'une formation, fut-elle courte, que la transmission contienne des éléments de l'analyse de pratiques effectuée dans le GAP ?

Il semble donc que la mixité des objectifs et des modalités de formation dans un GAP interroge la place que peut y avoir la modalité particulière qu'est l'analyse de pratiques en tant qu'outil de formation.

\section{L'analyse des pratiques en formation des enseignants : quels usages pour les GAP?}

\subsection{Une notion floue, des usages divers}

Les dispositifs d'analyse de pratiques mis en place en formation des enseignants, qu'elle soit initiale ou continue, sont extrêmement variées tant du point de vue des finalités poursuivies, des cadres théoriques mobilisés, que des méthodologies employées (Marcel, Olry, Rothier-Bautzer \& Sonntag, 2002).

Bronckart (2010) situe l'émergence de l'analyse de pratiques dans les dispositifs de formation d'enseignants aux confluences de la diffusion du mouvement du «praticien réflexif » (Schön, 1983) et du développement de techniques d'analyse du travail dans le champ de la formation pour adultes. Ainsi, le modèle du «praticien réflexif » postule qu'un praticien dispose de savoirs tacites, pratiques qu'il mobilise en cours d'action pour réguler une situation et que ces savoirs sont accessibles à condition de proposer un cadre où le praticien pourra verbaliser sa réflexion sur l'action vécue, en tant que « relecture de l'expérience » (Perrenoud, 2001). Ainsi, « on peut définir un dispositif d'analyses de pratiques comme un espace de formation réunissant les conditions pour qu'un sujet puisse opérer un retour réflexif sur sa pratique professionnelle, et comprendre ce qui se joue dans la complexité des situations difficiles et des moments de réussite » (Faingold, 2006). 
Différentes techniques sont alors mises en œuvre pour créer ces «conditions» de verbalisation. Schématiquement, deux types de techniques peuvent être distingués : des discours sur les pratiques et l'observation des pratiques (Marcel et al., 2002).

\subsection{L'analyse de pratiques en formation d'enseignants}

Dans ce cadre, l'enseignant est posé en "médiateur de la connaissance de ses pratiques » (Marcel et al., 2002), dans le sens où c'est lui qui décrit, hors contexte, à la fois la situation et son action, telles qu'il les a perçues sur le moment.

Différentes modalités sont alors possibles. La formation peut se centrer sur :

- Des entretiens d'explicitation (Vermersch, 1994) dans lesquels l'action vécue est verbalisée par l'enseignant et élucidée dans le discours par exemple par un passage du registre du «pré-réfléchi » à celui du « réfléchi ».

- L'auto-confrontation (Clot, 2001), dans laquelle un enseignant est amené à observer puis débattre d'une situation réelle et filmée de sa propre activité. L'auto-confrontation « engendre un débat autour des conditions de réalisation du travail, qui fait émerger une nouvelle expérience de l'activité » (Bronckart, 2010).

- L'instruction au sosie (Clot, 2001) dans laquelle un enseignant doit donner des instructions à un sosie chargé de le remplacer au pied lever. Il s'agit alors d'une « reconstruction de significations à propos de l'activité et du métier, mais sous une modalité planificatrice» (Bronckart, 2010).

Alors que les techniques fondées sur le discours sur les pratiques s'intéressent à une verbalisation d'enseignants sur les pratiques, les observations des pratiques centrent l'analyse sur ce qu'il se passe effectivement dans les classes, à l'aide de séances filmées.

Différents cadres théoriques peuvent être utilisés pour analyser l'action ou l'activité effectives :

- La Théorie de l'Action Conjointe en Didactique (Sensevy, 2011), qui en tant que théorie du didactique, centre l'analyse sur le savoir 
enseigné, à la fois en tant qu'objet des interactions entre l'enseignant et les élèves et objet d'apprentissage. Elle propose une modélisation de l'action en classe en tant que jeu entre l'enseignant et les élèves, dont l'enjeu est un savoir.

- La didactique professionnelle vise à « rendre compte des processus qui sont en jeu d'une part, dans la formation des compétences lors des situations dans lesquelles intervient une intention de formation et, d'autre part dans le développement de ses compétences au cours de l'activité de travail » (Rogalski, 2004). Elle se centre davantage sur l'analyse de la tâche plutôt que sur celle de savoirs académiques. Elle intègre au cadre classique de l'activité une approche développementale, fondée sur les travaux de Piaget et Vygotski.

- Le cours d'action postule, en s'appuyant notamment sur les travaux de Varela, que l'activité humaine est un élément du couplage structurel entre un individu et une situation. Ce couplage se spécifie à la fois par une reconnaissance dans la situation de ce qui fait signes pour l'acteur (via ses expériences passées) et par une interaction avec la situation. L'expérience de l'acteur est alors reconstruite en créant à la fois les conditions de son explicitation par l'acteur et une observation de son action (Theureau, 2006).

$\mathrm{Si}$ des dispositifs de formation utilisent souvent les discours des enseignants comme moteur de la formation, peu d'articles relatent à notre connaissance des modalités de formation fondés sur l'observation effective des enseignants en formation, à partir d'un cadre théorique issu des sciences de l'éducation.

\subsection{L'analyse de pratiques dans les GAP : le choix d'une perspective didactique}

D'un point de vue intrinsèque au GAP, nous avons vu que deux objectifs sont conduits simultanément : produire des ressources pédagogiques testées en classe autour d'un thème (majoritairement de type disciplinaire) et former des enseignants à leur transfert à d'autres enseignants (former des formateurs d'enseignants). La poursuite simultanée de ces deux objectifs institue donc l'enseignement/apprentissage de savoirs particuliers au centre du dispositif, aussi bien dans ce qui constitue le cœur des échanges du collectif, que de ce 
qui est transféré en formation, et en ce sens, on peut dire qu'il s'agit d'un dispositif de formation didactique.

L'analyse de pratiques dans le cadre d'un GAP relève d'une analyse de pratiques didactique (Orange, 2006). La place centrale accordée à l'enseignement/apprentissage de savoirs particuliers fait que le primat de l'analyse porte sur l'activité intellectuelle des élèves. La difficulté de l'analyse des pratiques est alors de rendre compte de cette activité.

Orange (2006) distingue deux pièges de ce type d'analyse de pratiques. Il est en effet commode d'évaluer l'activité intellectuelle des élèves d'une part dans l'écart à la norme académique (les élèves savent ou ne savent pas par rapport à ce qu'ils sont sensés savoir à la fin de la séance) et d'autre part dans l'écart par rapport à la réalisation d'une tâche prescrite (les élèves font ou ne font pas par rapport à ce qu'ils sont sensés faire dans la fin de la séance). Ces deux « commodités » sont des pièges pour Orange (2006), car «toutes deux fonctionnent dans la confusion entre la performance (seule visible dans les productions d'élèves) et l'apprentissage. Ce sont des attracteurs puissants, qui guettent en permanence le plus « réflexif » des enseignants. Ils font obstacle à une analyse didactique : la capacité de les éviter et les dépasser marque la rupture didactique qui est nécessairement en jeu dans toute analyse de pratiques didactique $»$.

Il nous semble donc que la rupture didactique souhaitée par Orange (2006) peut être un élément de la professionnalisation des enseignants du GAP et, en tant que tel un indicateur de développement dans la posture de l'enseignant membre du GAP - futur formateur d'enseignants.

\section{D'enseignant à formateur d'enseignants, le GAP comme espace de développement professionnel ?}

Amener les enseignants à la rupture didactique nécessite pour les animateurs du GAP « des repères théorico-pratiques sur les moyens d'aider les enseignants en formation à dépasser cet obstacle » (Orange, 2006). On peut donc interroger ces repères, notamment dans le lien qu'ils ont avec les savoirs académiques produits par les sciences de l'éducation, et leurs effets sur le développement professionnel des enseignants.

\subsection{La recherche en sciences de l'éducation comme facteur de développement professionnel des enseignants}


S'intéresser au développement professionnel des enseignants, c'est tenter de comprendre comment leur savoirs évoluent dans leur pratique. Le développement professionnel peut ainsi être conçu du point de vue du déroulement professionnel du sujet (au travers par exemple de la formation initiale, de l'entrée dans la profession, de la consolidation des compétences, de l'évolution de la profession). Mais il existe aussi une autre approche plus «professionnalisante», qui aborde le développement professionnel comme un processus d'apprentissage. Il s'agit alors d'un «processus par lequel, individuellement et collectivement, les enseignants révisent, renouvellent et augmentent leur engagement en tant qu'agents de changement, aux fins morales de l'éducation. Grâce à ce processus, ils acquièrent et développent de façon critique le savoir, les habiletés et l'intelligence émotionnelle qui sont essentiels à une pensée, à une planification et à une pratique de qualité, tout au long de la vie professionnelle $»($ Day, 1999).

Ce processus de développement professionnel s'envisage ainsi avec une visée de transformation et d'évolution des pratiques, ou bien avec une visée de production de connaissances, mais on peut aussi émettre l'hypothèse d'une dialectique entre ces deux visées.

Cette dialectique est d'autant plus crédible que le développement professionnel s'inscrit dans une dynamique de recherche. En effet, la recherche peut participer de manière efficiente au développement professionnel, comme c'est le cas dans les actions de formation par la recherche. Ceci suppose de maintenir des exigences épistémologiques comme le suggère Bru, c'est à dire " d'identifier des régularités ou des stabilités qui permettent de caractériser les savoirs et les pratiques construits et mobilisés par des professionnels puis de comprendre et d'expliquer leur dynamique évolutive »(Bru, 2002). En ce sens la recherche peut participer au développement des acteurs, à partir de la définition d'une problématique professionnelle, en les accompagnant dans l'analyse de leurs pratiques via des cadres théoriques et méthodologiques issus des travaux académiques, ce qui contribue au développement de leurs ressources cognitives, de leurs compétences par les pratiques d'échanges et de collaborations. On peut donc considérer « le développement professionnel [comme] un processus de changement, de transformation, par lequel les enseignants parviennent peu à peu à améliorer leur pratique, à maîtriser leur travail et à se sentir à l'aise dans leur pratique » (Uwamariya et Mukamurera, 2005). 


\subsection{Indicateurs du développement professionnel des enseignants}

Des indicateurs du développement professionnel ont été définis en rapport avec les différentes approches évoquées ci-dessus: il s'agit d'indicateurs conceptuels qui caractérisent les savoirs des acteurs en situation d'activité professionnelle et de formation, seul ou collectivement. Ces indicateurs visent à partir des individus eux-mêmes à décrire et comprendre les processus cognitifs ainsi que l'évolution des représentations. Ces deux niveaux, processus cognitifs et représentations, peuvent être perçus au travers notamment d'un repérage et d'une analyse des informations fonctionnelles qui guident l'action. Mais cela implique d'élaborer des processus de conceptualisation au travers de démarches compréhensives. On peut distinguer la conceptualisation qui se construit dans l'action et la conceptualisation qui se construit après l'action au travers d'une analyse spécifique ou d'un retour réflexif sur l'action, individuelle ou collective. Le développement professionnel peut s'appréhender à partir de l'étude de ces deux types de conceptualisations et de leurs évolutions. Ces conceptualisations, pour les enseignants, peuvent favoriser la construction de savoirs professionnels notamment au travers d'une participation à la conception et la mise en œuvre d'analyses de pratiques et de dispositifs pédagogiques et didactiques (ingénierie coopérative).

L'étude des ressources produites par les sujets ainsi que les usages d'artefacts ou d'instruments, au sens d'artefact et de schème d'utilisation associé (Rabardel, 2005) peuvent également constituer des indices du développement professionnel.

Un autre indicateur nous paraît pertinent dans cette démarche compréhensive du développement professionnel, c'est la focale sur le jugement qu'effectue le sujet à partir d'informations diverses ainsi que sa capacité à traiter ces informations pour appréhender la complexité des tâches, les analyser et trouver les solutions pour réduire cette complexité.

Enfin il nous parait important de ne pas omettre la construction collective des conceptualisations qui permettent de diagnostiquer avec acuité les situations, d'orienter l'action et surtout de communiquer ou de transmettre ces concepts et les évolutions de l'action envisagées soit à l'intérieur d'un groupe professionnel soit plus largement. Dans ce processus on peut également noter ce que Clot \& Faïta (2000) nomment le genre professionnel qui en tant qu'indicateur du développement professionnel permet de cerner 
les conceptualisations implicites construites dans des groupes de travail, et qui peuvent déboucher sur des compétences collectives, voire une mémoire collective, c'est à dire la capacité qu'ont les individus à collaborer pour créer des ressources cognitives. Le développement professionnel ne peut s'entendre hors de « la restructuration des significations, qui est le gage d'un réel développement personnel, procède de l'intériorisation et de la confrontation de ces différentes figures possibles de l'action; qu'elle consiste en la mise en œuvre d'une dynamique interprétative interne, qui «réfracte» la dynamique externe (sociale) des interprétations de l'activité humaine. La mise en place d'appuis formateurs visant à la construction, chez les formés, de cette confrontation dynamique, constitue donc, à nos yeux, une condition majeure pour que les prises de conscience issues de l'analyse des pratiques entraînent une restructuration du psychisme » (Bulea \& Bronckart, 2010).

\section{Questions de recherche}

Les changements de postures et de fonctions ne vont pas de soi et impliquent à la fois des remises en cause des pratiques ordinaires des enseignants et l'acquisition de savoirs et compétences singulières autorisant de nouvelles postures pour s'adapter aux besoins d'un public de pairs. Si un certain nombre de recherches en sciences de l'éducation ont abordé la question du développement professionnel des enseignants comme évoqué cidessus, peu à notre connaissance l'ont fait au regard du passage d'enseignant à formateur. Parallèlement les analyses de pratiques se sont développées dans la formation des enseignants mais en utilisant principalement des dispositifs de discours sur les pratiques. Nous nous intéressons ici aux analyses de pratiques didactiques, donc centrées sur la question des savoirs (savants, à enseigner, enseignés) en circulation dans les actions entre enseignants et élèves comme base potentielle de construction de compétences de formateur. Il s'agit donc de s'interroger, sur le rôle potentiel de l'analyse des pratiques didactique dans le changement de posture et de fonctions des enseignants devenant formateur, notamment si l'on considère que «l'analyse des pratiques ne peut constituer une démarche indépendante (voire opposée) à celle de l'acquisition de connaissances théoriques; au contraire, son but essentiel est de ré-interroger ces mêmes connaissances théoriques, et de leur faire prendre corps dans le cadre des pratiques didactiques effectives » (Bulea \& Bronckart, 2010). L'analyse de pratiques peut-elle alors participer, pour ces enseignants à leur développement professionnel de «formateur» d'enseignants? Quels types de savoirs, 
épistémologiques et didactiques, intègre un enseignant pour devenir formateur? Comment ces enseignants peuvent-ils construire les connaissances nécessaires au rôle de formateur et les transmettre ? A quels niveaux les analyses de pratiques peuvent-elles être utilisées et comment ?

Nous proposons dans ce chapitre une étude exploratoire à partir du travail de deux GAP qui fonctionnent depuis 4 années : le GAP documentation et le GAP physique-chimie.

\section{Le cas du GAP Documentation et du GAP Physique-Chimie}

Nous illustrons dans cette partie l'analyse didactique de pratiques d'enseignement telle qu'elle a été choisie et menée dans les deux GAP Documentation et Physique-Chimie, en centrant l'analyse sur les indices de développement professionnel que sont la construction collective des conceptualisations, les processus de conceptualisation et les ressources produites au sein des GAP. La didactique est entendue ici au sens d'analyser, de décrire et d'expliquer l'action conjointe un situ du professeur et des élèves par rapport à des enjeux de savoir et ce dans une double logique, celle interactive des contraintes et des possibles de la relation didactique et celle épistémologique du «contrôle» des savoirs mis à l'étude au travers d'une analyse à priori au regard d'une théorie du savoir enseigné (Amade-Escot, 2007).

\subsection{Le Gap documentation}

\subsubsection{Modalités de travail collectif}

L'approche collective développée au sein du GAP documentation s'est organisée autour d'un groupe de 10 professeurs-documentalistes volontaires et s'est progressivement centrée sur une méthodologie d'analyse didactique des pratiques d'enseignement en regard des difficultés repérées dans l'enseignement de notions nouvellement inscrites dans les référentiels de formation. La première étape a consisté à construire collectivement une séance de cours sur le concept de document à partir des préconisations des programmes. Cette première séance a été réalisée par un enseignant, filmée et entièrement retranscrite puis analysée collectivement par le groupe. Elle présentait un découpage du savoir enseigné basé sur la mobilisation des conceptions initiales des élèves autour de l'aspect définitoire de la notion de 
document. Les exercices proposés consistaient à mettre en relation des termes contribuant à une première définition de la notion. Nous avons donc procédé à une analyse didactique de la séance à partir des questions suivantes : quels savoirs enseignés ? quelles pratiques d'enseignement? quelles situations pour les savoirs ? Pour cette analyse nous avons utilisé un certain nombre de descripteurs issus de la théorie de l'action conjointe en didactique (TACD) dans l'objectif d'acquérir collectivement des outils d'analyse permettant de décrire, de comprendre et d'expliquer l'action. Nous proposons de présenter ci-dessous rapidement les principaux éléments utilisés.

\subsubsection{Cadre d'analyse et processus de conceptualisation}

La TACD considère les échanges didactiques comme des transactions à propos des savoirs. Les savoirs sont donc vus comme des objets transactionnels et la TACD s'applique à décrire ce que fait chacun des transactants avec ces savoirs. La TACD s'appuie sur le modèle du jeu didactique qui permet de décrire l'action didactique. Le jeu didactique est un jeu coopératif dans lequel l'élève doit s'investir et au cours duquel le professeur doit faire preuve de retenue et ne rien dévoiler qui amènerait l'élève à produire des stratégies gagnantes de faible valeur. Il s'agit avant tout d'un jeu de savoir, impliquant l'appropriation effective d'un savoir dans lequel l'élève doit mettre en place une stratégie gagnante de son propre mouvement.

Dans la TACD, les jeux d'apprentissages constituent des moments repérables dans le déroulement d'un cours. Leur succession répond à une nécessité d'avancer dans l'apprentissage et par un nouvel enjeu de savoir. Ils sont caractérisés par quatre types de descripteurs. Définir réfère à ce que fait le professeur pour que les élèves sachent précisément à quel jeu ils doivent jouer. Dévoluer désigne ce que fait le professeur pour que les élèves prennent la responsabilité de leur travail. Réguler définit ce que fait le professeur en vue d'obtenir une stratégie gagnante de la part des élèves. Institutionnaliser comprend ce que fait le professeur pour que tel comportement ou connaissance soit légitime, et attendue dans l'institution classe. Ces quatre catégories permettent de comprendre les modalités de la construction des savoirs dans la classe.

Les jeux d'apprentissage constituent une entité, un ensemble d'éléments d'un même savoir présents dans un même contrat et un même milieu. 
Autrement dit définir «un jeu d'apprentissage consiste donc à utiliser conjointement deux descripteurs : le milieu et le contrat associés à un enjeu de savoir déterminé»(Sensevy, 2011). Ce dernier définit le contrat didactique comme un système d'habitudes engendrant lui même un système d'attentes mais aussi un système de normes pour un jeu particulier., autrement dit il s'agit d'un «système stratégique disponible au moyen duquel le professeur et les élèves vont jouer ce jeu »(Sensevy, 2011). Il distingue dans le contrat une composante épistémique et une composante transactionnelle. Le milieu est lui approché du point de vue de l'environnement de l'action, il comprend la situation, les stratégies qu'il permet de développer, il est donc un potentiel d'action qui sert à faire des expériences didactiques en permettant à 1 'élève de faire les connexions nécessaires à l'élaboration de la pensée.

Des descripteurs de l'action conjointe en didactique sont définis pour décrire, expliquer et comprendre les enjeux de savoirs en classe. Ainsi la chronogenèse permet d'étudier l'avancée des savoirs sur l'axe du temps : avancée, accélération, ralentissement, stagnation, disparition. La topogenèse cherche à décrire le partage des responsabilités dans les transactions didactiques c'est-à-dire l'évolution des responsabilités que chacun des acteurs, professeur ou élève, prend en charge par rapport à l'objet de savoir mis à l'étude. La mésogenèse décrit l'action sur le milieu didactique, c'est-àdire l'évolution du système d'objets, qu'ils soient matériels, symboliques ou langagiers, qui délimitent le contexte de l'action du professeur et des élèves. Ces outils de description permettent d'établir un point de vue sur l'action didactique intentionnelle considérée comme une action conjointe ternaire entre professeur, élève et savoir. L'usage de ces descripteurs a constitué pour les professeurs-documentalistes du GAP un premier niveau de conceptualisation sur leurs pratiques.

\subsubsection{Processus cognitifs et évolution des représentations}

Concrètement, l'analyse, notamment chronogénétique, a permis de pointer le peu d'avancée des savoirs au cours de la séance ce qui a débouché sur l'importance d'écrire un " texte de savoir », c'est à dire ce que l'élève doit apprendre en référence à la conception scientifique d'un objet de savoir. En effet certains savoirs restent très approximatifs et on retrouve des erreurs dans la manière de les formuler. 
Le domaine de validité (contexte d'utilisation) et le statut des savoirs des savoirs ne sont pas précisés. L'importance de la référence au réel dans son lien avec le modèle plus abstrait est absente, de même que l'association des mots et des pratiques. Nous avons cependant noté des «marquages » dans le discours de l'enseignant qui introduisent un apport. Il semble alors qu'il s'agit de passer de relations syntaxiques (abstraction, idée, « grammaire » scientifique) aux relations avec le réel (les pratiques documentaires), mais en omettant les relations sémantiques, c'est à dire ce qui donne sens entre les deux souvent de l'ordre de l'implicite dans les interactions analysées. L'analyse a également mis au jour l'importance du milieu pour faire avancer les savoirs, ainsi que la place du contrat didactique qui régit fortement les transactions.

Les résultats de l'analyse de la séance synthétisés ici montrent qu'un processus cognitif collectivement partagé s'est construit progressivement et ce grâce à la mobilisation de référents théoriques, faisant évoluer les représentations initiales. Ainsi le peu d'avancée des savoirs, l'absence d'institutionnalisation, le maintien dans les conceptions du quotidien des élèves au détriment d'une avancée de l'histoire scientifique pourtant objectivée dans la préparation, a fait prendre conscience aux enseignants engagés dans cette réflexion d'une part d'une non maîtrise conceptuelle des savoirs enseignés et d'autre part d'une inadéquation du milieu avec les objectifs pédagogiques et didactiques.

\subsubsection{Ressources produites et constructions collectives de conceptualisation}

A partir de cette analyse, le professeurs-documentalistes ont collectivement reconstruits une nouvelle séance pédagogique qui montre comment l'usage des différends descripteurs didactique a permis de faire évoluer les représentations initiales des enseignants.

Un retour sur le savoir savant a d'abord été nécessaire et s'est concrétisé par la suite par l'écriture collective d'un texte de savoir sur le document. Celui-ci a guidé la reconstruction de la séance. On peut dire ici que les ressources produites collectivement ont participé de cet effort de conceptualisation et contribuent globalement à une certaine forme de développement professionnel des enseignants membres du GAP. L'étape suivante qui a consisté à préparer des séances de formation dites de démultiplication pour leurs collègues en région, a confirmé l'importance 
d'une analyse didactique des pratiques théoriquement instrumentée en regard d'un changement de posture, même si les enseignants ont souligné que cette analyse didactique si elle est primordiale pour eux ne peut être reproduite en l'état sans l'accompagnement dans la durée de référents comme c'est le cas au sein du GAP. Autrement dit, si on peut avancer l'hypothèse d'un développement professionnel potentiel pour les enseignants membres du GAP basé sur des processus cognitifs de conceptualisation, d 'évolution des représentations au travers des ressources collectivement produites, on ne peut postuler en l'état d'un possible élargissement de ce développement à tous les enseignants car il nécessiterait la même démarche d'apprentissage basé sur une appropriation de cadre théorique au service de l'analyse et de l'évolution des pratiques.

\subsection{Le Gap Physique Chimie}

\subsubsection{Modalités de travail collectif}

Le GAP de Physique Chimie est structuré en 3 groupes explorant chacun l'enseignement d'un thème particulier: l'énergie, les biomolécules et la modélisation. Ces 3 groupes, constitués de 3 enseignants volontaires, sont encadrés par 3 personnes différentes : deux formateurs et un chercheur en didactique de la physique (groupe modélisation). Le dispositif alterne séminaires, visioconférence et travail autonome des enseignants dans leur établissement. Deux séminaires sont organisés par an dans lesquels les enseignants du GAP travaillent au sein de chaque groupe à la production de ressources ou à la construction d'une formation, et ils présentent leurs travaux au reste du GAP. Les ressources produites sont ensuite testées en classe et leur mise en œuvre est discutée lors du séminaire suivant.

Les 3 groupes ont ainsi travaillé de manière identique et la méthode privilégiée pour analyser les pratiques d'enseignement a été l'entretien d'explicitation portant sur la mise en œuvre en classe des ressources construites en groupe. Les échanges ont porté sur l'appropriation individuelle du travail collectif à travers les fiches pédagogiques construites par les enseignants (adaptées au contexte de leur établissement et de leurs classes), ainsi que sur la présentation de quelques travaux d'élèves.

\subsubsection{Cadre d'analyse et processus de conceptualisation}


Aucun élément théorique n'a été amené par les animateurs du GAP pour conceptualiser les pratiques et en permettre l'objectivation.

Des indices de développement sont tout de même différents suivant les groupes. Pour les groupes travaillant sur l'enseignement des biomolécules et de l'énergie, le travail a porté uniquement sur ces thèmes et les discours explicités portent sur la description de la mise en œuvre des ressources produites. L'animation des groupes s'est faite « entre pairs », dans le sens où les savoirs à enseigner ont été interrogés sans faire intervenir de perspectives extérieures à leur enseignement (points de vue académiques ou des sciences de l'éducation par exemple).

Pour le groupe sur la modélisation, on observe que si l'explicitation des discours porte également sur la mise en œuvre des ressources collectives produites, des apports ont été faits par l'animateur du groupe sur l'épistémologie des sciences et des savoirs scientifiques scolaires.

On note donc ici une différence entre les groupes: si aucun cadre théorique sur l'analyse des pratiques n'a été travaillé avec les enseignants, il y a eu utilisation d'un cadre théorique issu de l'épistémologie et de la didactique des disciplines scientifiques dans un des groupes.

\subsubsection{Les ressources produites}

Les 3 groupes ont produit des ressources pédagogiques (travaux pratiques, activités, etc.) et ont également construit et mis en œuvre une formation en région d'une journée à leurs collègues. Les productions du groupe «modélisation» se distinguent de celles des groupes «biomolécules » et énergie » sur deux points. Tout d'abord, les enseignants du groupe modélisation ont étendu individuellement à d'autres objets de savoir le travail fait en groupe lors des séminaires : ils ont été ainsi capables de « transférer » ce qu'ils ont mis en place au sein du GAP à d'autres savoirs à enseigner, et ont ainsi poursuivi la production de ressources pédagogiques. Ensuite, ils ont également produit une ressource "méta », à destination exclusive des enseignants, sur l'épistémologie associée à la modélisation et à son enseignement. Autrement dit, contrairement aux deux groupes précédents, ils ont été capables de conceptualiser ce qu'ils ont mis en œuvre dans le GAP et avec leurs classes. 


\section{D'enseignant à formateur d'enseignant : un dispositif vecteur de changement}

Nous reprenons ici successivement les questions de recherche que nous avons posées dans la partie 5 .

\subsection{Les savoirs pour devenir formateur}

Pour Clement et Vandenberghe (1999), le développement professionnel des enseignants s'apparente à un processus d'apprentissage et l'enseignant est similaire à un apprenant qui, au fil du temps et de ses expériences, construit des savoirs professionnels, qui intègrent à la fois des savoirs théoriques (disciplinaires, didactiques, pédagogiques, etc.) et des savoirs pratiques (issus de l'expérience et de la réflexion) (Uwamariya \& Mukamurera, 2005).

Dans le cadre du GAP de Documentation, nous avons vu que les sciences de l'éducation ont une place privilégiée, à la fois comme outils conceptuels et méthodologiques pour analyser les pratiques d'enseignement filmées, mais aussi en tant que révélateurs (au sens photographique du terme) des savoirs savants à enseigner, qui sont ainsi réinterrogés collectivement.

Dans le GAP de Physique-Chimie, ce sont les savoirs pratiques qui sont les objets de l'analyse, et dans le cas des groupes sur les biomolécules et l'énergie, les savoirs savants ne sont questionnés que dans le périmètre de ces savoirs pratiques. Dans le groupe sur la modélisation, des savoirs théoriques de type épistémologique sont placés au centre des préoccupations des enseignants.

\subsection{Un dispositif de développement professionnel}

Les deux GAP fonctionnent comme des communautés de pratique (Lave $\&$ Wenger, 1991), dont un des principes fondateurs est que « l'apprentissage et le développement se produisent lorsqu'une personne participe aux activités de sa communauté transformant ainsi sa compréhension, ses rôles et ses responsabilités au fil de sa participation » (Rogoff, Matusov \& White, 1996). Ainsi, l'apprentissage des savoirs professionnels au sein de ce dispositif se situe entre les pratiques sociales de la communauté GAP et les processus de construction individuelle (Mottier Lopez, 2008). 
Nous avons vu dans les deux exemples décrits précédemment que si les savoirs pratiques associés à l'enseignement de savoirs particuliers sont au fondement des échanges entre les enseignants, les éléments les plus apparents sur lesquels se construit leur professionnalisation sont des éléments issus des sciences de l'éducation et de l'épistémologie de leur discipline. En ce sens, il y a non seulement une préoccupation commune (liée aux savoirs pratiques), mais également un apprentissage explicite commun (lié à des sciences académiques), qui permet d'éclairer différemment les savoirs à enseigner.

\subsection{A quels niveaux les analyses de pratiques peuvent-elles être utilisées et comment ?}

En nous intéressant au fonctionnement de ces deux GAP disciplinaires, il nous semble qu'une des particularités des analyses de pratiques effectuées est que celles-ci, quand elles s'appuient sur des cadres théoriques de type didactique et/ou épistémologique conduit les enseignants d'une part à davantage s'approprier le passage des savoirs savants aux savoirs enseignés et d'autre part en conceptualisant à se développer voire à changer de posture.

Si les dispositifs mis en place dans ces deux GAP nous paraissent intéressants en terme de dispositifs de développement professionnel appuyés sur des analyses didactiques des pratiques, il n'en reste pas moins que la question de la place de l'analyse didactique de pratiques au sein des sessions de formation régionale ou interrégionale reste entière pour l'institution. En effet même dans l'hypothèse d'un changement de posture pour ces enseignants, nous avons relevé que le cheminement effectué grâce à ces analyses didactiques ne peuvent se faire que dans la durée et accompagnée par des experts en capacité d'introduire les savoirs nécessaire au delà de la simple réflexion sur la pratique.

\section{Conclusion}

Si le dispositif GAP nous paraît constituer un vecteur de changement en formation continue des enseignants, en ce sens qu'il permet de lier l'analyse réflexive à des processus de conceptualisation appuyés sur des savoirs, on peut dire qu'il constitue également un moteur pour le développement professionnel de ces enseignants. Il paraît en effet important de maintenir une exigence épistémologique en identifiant des régularités ou des stabilités 
qui permettent de caractériser les savoirs et les pratiques construits et mobilisés par des professionnels puis de comprendre et d'expliquer leur dynamique évolutive (Bru, 2002). Cependant l'analyse didactique n'épuise pas l'analyse des pratiques, dans notre cas cette focale résulte du choix organisationnel d'un GAP disciplinaire (il existe par ailleurs d'autres types de GAP à visée plus transversale). Mais le développement professionnel signifie une sorte de recherche continuelle faite par l'enseignant sur sa propre pratique (Lieberman et Miller, 1990). L'enseignant étudie, analyse et évalue régulièrement ses activités pour être en mesure de relever les défis auxquels il fait quotidiennement face. L'amélioration de la pratique, la maîtrise du travail enseignant et l'évolution sont des actions qui impliquent pour l'enseignant un examen critique des faits éducatifs et de sa pratique. L'idée commune qui ressort est que le développement professionnel est un processus de changement, de transformation, par lequel les enseignants parviennent peu à peu à améliorer leur pratique, à maîtriser leur travail et à se sentir à l'aise dans leur pratique (Uwamariya, A. \& Mukamurera, J., 2005). Cette approche du développement professionnel s'appuie sur une certaine forme de rapport au savoir pour les professionnels, qui gagne à être analysé sous l'angle du système didactique.

L'institution crée dans le cas des GAP un contexte de changement pour les enseignants participant au dispositif, qui nous paraît donc vecteur de changement, de par l'enrichissement de la posture réflexive par un outillage théorique académique. Pour autant le changement de posture espéré vers un rôle de formateur en capacité de reproduire pour d'autres ce même cheminement reste plus aléatoire même si la somme des connaissances mises en commun sur lesquelles les acteurs peuvent avoir une approche signifiante prend sens dans son application, c'est à dire inscrite dans une dynamique d'utilisation, de transmission et d'appropriation. Mais cette question nécessiterait une étude plus longitudinale qui permettrait d'affiner cette question qui a été ici posée de manière exploratoire. 